\title{
Forensic Data Hiding Optimized for JPEG 2000
}

\author{
Dieter Bardyn*, Johann A. Briffa ${ }^{\dagger}$, Ann Dooms*, Peter Schelkens* \\ *Dept. of Electronics and Informatics (ETRO), \\ Vrije Universiteit Brussel (VUB) \\ Interdisciplinary Institute for Broadband Technology (IBBT), \\ Pleinlaan 2, B-1050 Brussels, Belgium \\ ${ }^{\dagger}$ Dept. of Computing, \\ University of Surrey, \\ Guildford, Surrey, GU2 7XH, United Kingdom \\ j.briffa@surrey.ac.uk
}

\begin{abstract}
This paper presents a novel image adaptive data hiding system using properties of the discrete wavelet transform and which is ready to use in combination with JPEG 2000. Image adaptive watermarking schemes determine the embedding samples and strength from the image statistics. We propose to use the energy of wavelet coefficients at high frequencies to measure the amount of distortion that can be tolerated by a lower frequency coefficient. The watermark decoder in image adaptive data hiding needs to estimate the same parameters used for encoding from a modified source and hence is vulnerable to desynchronization. We present a novel way to resolve these synchronization issues by employing specialized insertion, deletion and substitution codes. Given the low complexity and reduced perceptual impact of the embedding technique, it is suitable for inserting camera and/or projector information to facilitate image forensics.
\end{abstract}

\section{INTRODUCTION}

The availability of cheap and accessible methods for recording and editing digital data makes the creation of digital reproductions simple and robust. Hence, there is great interest in developing technology that helps to protect the integrity of a digital work and the intellectual property rights (IPR) of its owners. This can be realized through watermarking, which imperceptibly alters the content to hide the required data. As JPEG 2000 [1] is the standard for digital cinema, and is becoming more and more popular for surveillance applications or archival objectives, it is natural to hide information in the discrete wavelet transform (DWT) coefficients used in the codec. This methodology eliminates the need for additional transforms and hence better integrates into existing systems.

Perceptual shaping is any technique that identifies coefficients which, when modified, cause the least amount of perceptible distortion. The use of perceptual shaping in data hiding improves performance in terms of lower perceptual distortion for fixed payload and robustness [2],[3]. This performance is due to the incorporation of how the human visual system processes visual stimuli by exploiting the image statistics. Indeed, most recent watermarking systems [4], [5] (based on the ideas of Costa's paper [6]), assume independent and identically distributed (i.i.d.) embedding samples in order to optimally use the available channel, which is difficult to realize in practice.

The most relevant perceptual shaping technique in our foreseen scenario is derived from the work of Lewis and
Knowles [7] which discusses the impact of quantizing certain wavelet coefficients. They determined an upper bound for the quantization stepsize to remain imperceptible. Their ideas were adopted by Barni et al. [3] in image-adaptive spread spectrum data hiding. Solanki et al. [2] on the other hand presented an approach which was far less complex and tuned for JPEG compression. In their paper, they identify 8 by 8 blocks of discrete cosine transform (DCT) coefficients which can tolerate the most distortion, both with respect to robustness and perceptibility. The energy of each block determines which ones can be modified and which can not. Our goal is to present a hybrid approach that has low complexity and perceptibility and moreover, is tuned for JPEG 2000.

The image adaptive selection of coefficients can cause additional errors, as the decoder has to estimate this selection from a modified source. Conventional error-correcting codes, assuming a substitution-only channel, are unusable under these conditions. Hence, specialized insertion, deletion and substitution (IDS) codes are required to correct these synchronization issues.

The paper is organized as follows. We start with describing the building blocks of our image adaptive data hiding system and how they interact. Along the way, we introduce our new mask and the novel combination witch IDS codes. In the following section we describe our test setup to investigate the performance of our system in terms of complexity, robustness and perceptibility, before we conclude with our final remarks in Section IV.

\section{THE BUILDING BLOCKS}

The presented image adaptive data hiding system can be split up into three major building blocks, consisting of perceptual shaping to minimize embedding distortion, quantization index modulation (QIM) [4] to actually hide information and IDS codes to tackle synchronization issues at the decoder side.

\section{A. Perceptual shaping}

We first describe the two existing perceptual shaping techniques on which our technique is based. The coefficients determined by these techniques are called to form a mask.

- An approach with low complexity was presented by Solanki et al. [2] which chooses coefficients to be mod- 
ified based on the energy of DCT coefficients of 8 by 8 blocks. Let $\left(c_{i j}\right)_{i j}=D C T_{2}\left(\left(a_{i j}\right)_{i j}\right)$, where $a_{i j}$ denotes the intensity values of an 8 by 8 block and $D C T_{2}$ is a two-dimensional DCT, then the energy is calculated as

$$
E_{\text {block }}=\sum_{i, j=0}^{7}\left\|c_{i j}\right\|^{2}-\left\|c_{00}\right\|^{2} .
$$

- Modifying certain wavelet coefficients results in spatial distortion spread over different pixels. For a wavelet decomposition with $L$ decomposition levels, $I_{l}^{\theta}$ denotes the subband at resolution level $l=1 \ldots L$ and with orientation $\theta \in\{0,1,2,3\}$. Lewis and Knowles [7] presented a mask which calculated for each wavelet coefficient $I_{l}^{\theta}(i, j)$ the maximal allowable distortion $q_{l}^{\theta}(i, j)$ based on detail level and orientation $\Theta(l, \theta)$, brightness $\Delta(l, i, j)$ and texture activity $\Xi(l, i, j)$.

$$
q_{l}^{\theta}(i, j)=\Theta(l, \theta) \Delta(l, i, j) \Xi(l, i, j)^{0.2} .
$$

Barni et al. [3] adopted this formula taking into consideration that the human eye is less sensitive to changes in very dark regions as well as to changes in very bright areas. We will refer to this mask as the Lewis-Barni mask.

- If we consider applying these schemes to a JPEG 2000 setup, it is clear that working in the same domain (i.e. wavelet) is of great interest. Furthermore, the complexity of the mask itself should not burden the applicability of the technique, especially with respect to real time applications. The Tree Based mask presents an approach with low complexity in the wavelet domain. Small modifications to a lower frequency wavelet coefficient will be masked if there is enough energy in the higher level frequency coefficients that will add detail to the spatial block from which that coefficient was determined. The higher frequency coefficients emanating from a lower frequency coefficient $I_{l}^{\theta}$ can be seen as a tree, whose energy $E_{T}$ is determined by

$$
E_{T}(l, \theta, i, j)=\sum_{k=l+1}^{1+a} \sum_{x=0}^{k-l} \sum_{y=0}^{k-l}\left\|I_{k}^{\theta}(i+x, j+y)\right\|^{2},
$$

where $a$ is a constant that eliminates some of the finer detail subbands to ensure proper robustness.

The obtained perceptual masks can then further be used to select which coefficients will be modified by using a threshold $T$. To keep the number of selected coefficients constant, we propose to dynamically determine the threshold using an iterative approach. If $(\rho(i, j))_{i j}$ represents one of the described masks, this approach calculates $M_{\alpha}=\left|\left\{\rho(i, j) \mid \rho(i, j)>T_{\alpha}\right\}\right|$ iteratively over $\alpha$ where $T_{0}=0$ and $T_{\alpha}=T_{\alpha}+1$. The iteration process is stopped when $M_{\alpha}$ is lower then the design payload size $\tilde{M}$. To ensure that all the information can be embedded we take the design payload size to be the required payload size $M$ plus $10 \%$.

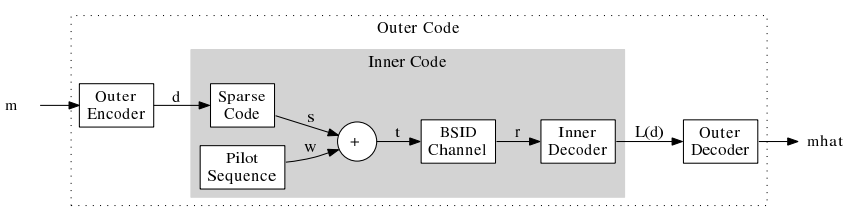

Figure 1. Structure of the Davey-MacKay concatenated code.

\section{B. Data Hiding}

In order to hide information we modify the selected coefficients using QIM [4]. This technique hides information through the selection of an appropriate quantizer. Since the data hiding system does not effect the comparative outcome for the different systems, we fix it to the commonly used binary scalar case. Here, a scalar quantizer $Q$, with stepsize $\Delta$, is employed to watermark the cover work $\mathbf{c}$ with message $m \in\{0,1\}$. To hide a message $m$, we quantize $\mathbf{c}$ using a dithered version $Q_{m}(\cdot)=Q\left(\cdot-\frac{m \Delta}{2}\right)+\frac{m \Delta}{2}$ of $Q$. The mask that operates in the DCT domain, uses a zigzag scan in the selected DCT blocks and modifies 8 coefficients in each block, so only $\frac{M}{8}$ blocks need to be selected. Solanki [2] used this technique for their most robust test setup. The data hiding techniques based on the masks that operate in the wavelet domain, modify all the selected coefficients.

\section{Insertion, Deletion and Substitution Codes}

The perceptual masking operation, which effectively controls perceptibility, causes desynchronization at the receiver. This can be modeled by an IDS channel operating in discrete time. Conventional error-correcting codes, assuming a substitution-only channel, are unusable under these conditions and very few practical codes exist for IDS channels. In this work, we use an improvement on the Davey-MacKay (DM) construction, originally presented in [8]; the improvements used here are those presented in [9], [10].

The DM system consists of three main components (see also Figure 1): an outer non-binary error-correcting code, a sparse code, and a pseudo-random binary pilot sequence. At the receiving end, the inner decoder estimates the most-likely protected message $d$ by comparing the received sequence with the pilot sequence and the additive sparse code at each symbol position. The likelihoods obtained are used by the outer decoder to estimate the message $m$.

1) Inner Codes: We restrict ourselves to the $(8,16)$ sparse inner code with balanced codebook, which has an effective code rate of $\frac{1}{2}$ and requires an outer code in GF (16). This code has been chosen because it is known to give good performance in channels with a high rate of insertions and deletions. We decode using the symbol-level decoder presented in [9].

2) Outer Codes: As outer codes, we use a parallel concatenation of non-binary constituent codes, as presented in [9]. The constituent code is defined over GF (16) by feedback polynomial $1+D+\alpha^{4} D^{2}$ and feed-forward polynomials $1+\alpha D+\alpha^{4} D^{2}$ and $1+\alpha^{2} D+\alpha^{9} D^{2}$, where $\alpha$ is a root of the primitive polynomial $x^{4}+x+1$. The turbo code uses 


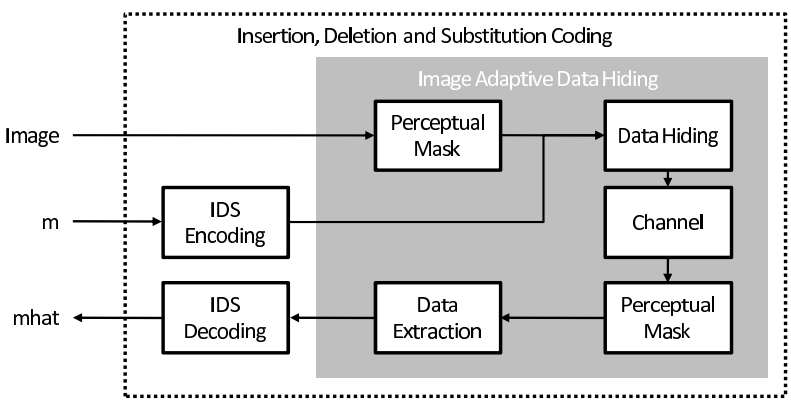

Figure 2. Overview of the proposed system.

an unterminated trellis, and an S-random interleaver [11] of block size $K=75$ and spread $S=7$.

3) Concatenated System: The combination of inner and outer codes specified above, results in a DM-turbo concatenated code with an input block size of $K=75$ symbols over GF (16) and an output block size of $N=3000$ bits.

\section{Complete System}

The different building blocks discussed in the previous section are combined into a complete system as shown in Fig. 2.

\section{RESULTS}

In this section we experimentally compare our newly developed mask with the Solanki and Lewis-Barni masks in terms of complexity, robustness and perceptibility in order to draw conclusions on the resulting image-adaptive data hiding systems. Robustness is measured by the amount of insertion and deletion errors that occur after common signal processing operations, while perceptibility will be measured by the peak signal to noise ratio (PSNR) and the structural similarity index measure (SSIM) [12].

For the results presented in this paper, we used the lossy 9-7 DWT transform, as defined in the JPEG 2000 coding standard, and decomposed each image in 4 wavelet levels. We determined the perceptual mask for level 3 of the decomposition based on the results for the LH filter, which showed the best performance in our experiments. For the Solanki mask, the setup is similar as in the original paper [2], with the exception that we dynamically calculated the threshold and did not apply JPEG compression while embedding. For the Tree Based mask we set the constant $a=1$ (see equation (1)). The techniques were tested on grayscale versions of AERIAL2, BIKE, CAFE, CATS, CHART, MAT, TOOLS, WOMAN, which are part of the set of test images for JPEG 2000 standardization and are assumed to accurately represent varying image types. Experiments on images of lower resolution and images with less texture (for example the image WATER from the same test set) showed that the Tree Based mask incurs more synchronization issues than the other two masks in this case. This is due to the fact that the number of coefficients which can be modified imperceptibly is far smaller than the number required to embed the entire payload. This means that, although the other two masks would be able to decode the hidden mark, the system would have failed anyway as the goal of imperceptible embedding would not be met.

The employed scalar QIM is designed to cause similar distortion in the modified spatial blocks, which is fixed at $52 \mathrm{~dB}$. With the described IDS construction, a 300-bit binary message (the actual information sequence) is first represented as a 75 -symbol string $m$ over GF (16). This is encoded by the DM-turbo code resulting in a 3000 -bit binary sequence, for an overall code rate $R=\frac{1}{10}$. Thus a fixed watermark payload of 3000 bits is be embedded in all images.

The decoder assumes the likelihood of insertion $P_{i}$ and deletion $P_{d}$ to be equal and fixed at $P_{i}=P_{d}=0.1$, while the likelihood of substitution $P_{s}=0$. This is justified because the likelihood of insertions and deletions (caused by loss of synchronization due to the perceptual mask) is significantly greater than that of substitutions (caused by failure of the watermark detector). While this assumption clearly gives some mismatch at the receiver, a reliable estimator has still not been developed for this channel. Unlike the simulations published in [9], we do not put a limit on the burst length $I$ of insertions, resulting in a small improvement on decoding performance. When applied to the output of the watermark decoder, we have observed that the best response is obtained if the received sequence is truncated to 3000 bits before decoding.

\section{A. Complexity of the masks}

As mentioned before, complexity should not be to high for a practical system. The Lewis-Barni mask uses 3 different factors to determine the masking coefficient and as such requires quite some computations. Without making claims about the optimization of the implementations used in our experiments, we saw that the Lewis-Barni mask took about 10 times more computation time than the other two masks.

\section{B. Robustness of the masks}

We compared the performance in terms of insertions and deletions, for both masks presented in the literature and the Tree Based mask introduced in this paper. Figure 3(a) shows the combined insertion-deletion (ID) likelihood $P_{i}+P_{d}$ for the different masks, as substitution errors are only caused by errors in the watermark detection, after JPEG 2000 compression at varying bits per pixel (bpp), after addition of AWGN for varying strengths and after JPEG compression at varying quality factors. The results show that the Tree Based mask does not perform as well as the masks used in this comparison, but at a maximum combined ID likelihood rate of 3\% (JPEG compression at quality factor 10) this is well within reach of the IDS codes employed in the second layer of our system.

\section{Data Hiding}

Detection is flawless for a PSNR between the watermarked and the distorted image down to $40 \mathrm{~dB}$, where the image was imperceptibly watermarked at $52 \mathrm{~dB}$ when all the coefficients were marked (so no substitution errors occur beyond this point). Distortions under consideration: addition of Gaussian 

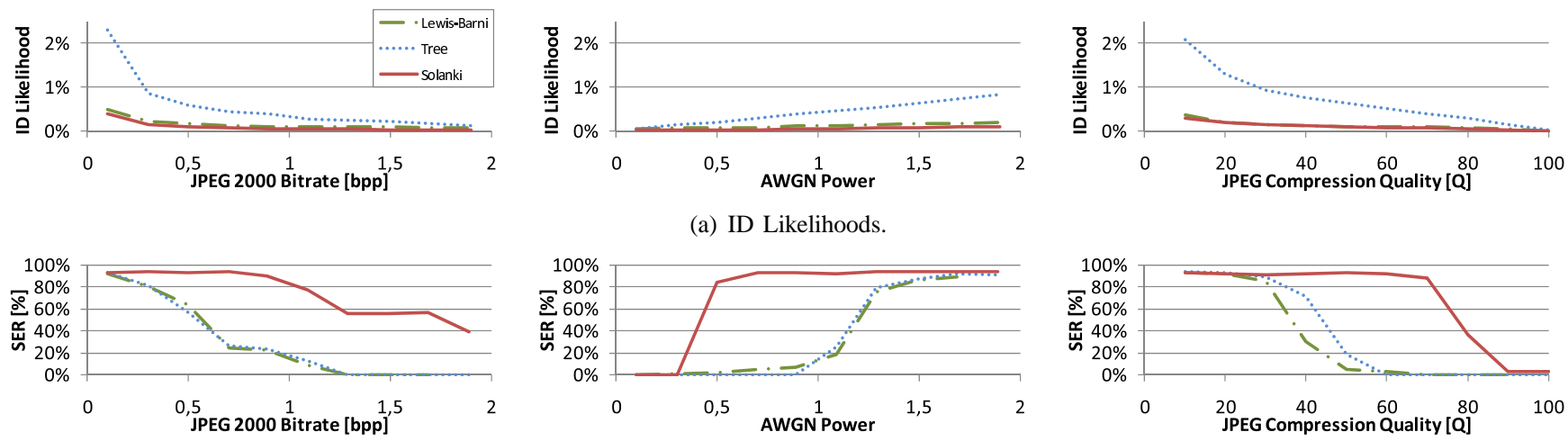

(a) ID Likelihoods.
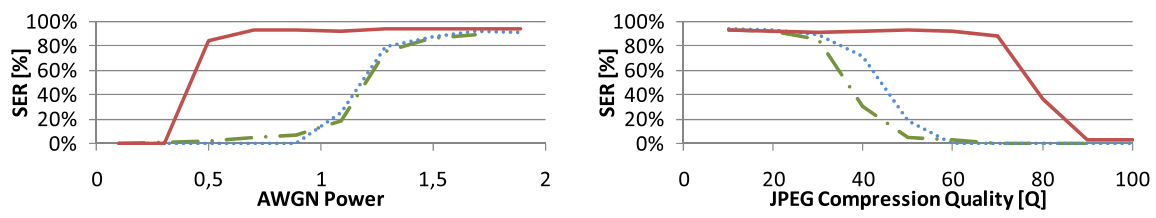

(b) Symbol Error Rates.

Figure 3. SER and ID Likelihoods after different signal processing operations.

noise to $40 \mathrm{~dB}$, JPEG 2000 compression down to 0.5 bpp and JPEG compression down to quality factor 70 . The technique that modifies DCT coefficients is far less robust and requires an embedding perceptibility of $40 \mathrm{~dB}$ to achieve the same goals.

\section{Perceptibility of the techniques}

To compare the visual impact of the three techniques, we measured the perceptibility by means of PSNR and SSIM for a watermark embedding distortion equal to the one used in the robustness tests (see Sect. III-E). The Solanki technique resulted in $55 \mathrm{~dB}$ and SSIM index of 0.9996. For this technique, watermarking the images CHART, MAT and TOOLS resulted in much higher perceptual impact, on average $30 \mathrm{~dB}$ PSNR and SSIM index of 0.9990. For the other techniques there was no noticeable difference over the image test set and averaged we measured a perceptibility of $59 \mathrm{~dB}$ between the original and the watermarked versions, while the SSIM index was perfect at 1.0000 .

\section{E. Complete system}

The overall robustness of the system is measured by comparing the decoded sequences and the original data, which consists of 300 symbols over GF (16), by means of symbol error rate (SER). Figure 3(b) shows the results, which indicate that the Tree Based technique performs similar as the Lewis-Barni technique in terms of robustness, but it clearly outperforms the other masks in terms of complexity (LewisBarni) or perceptibility (Solanki).

\section{CONCLUSION}

This paper presents a novel watermarking system ready for use in a forensic data hiding environment. The technique operates on wavelet transform coefficients to determine a perceptual mask and to embed the desired payload. Two components, a new perceptual shaping technique and stateof-the-art IDS codes, are combined in an innovative way to successfully solve the problem of desynchronization that occurs when using perceptual masks. The new perceptual shaping technique is far less complex than existing techniques and, when combined with the proposed error correcting layer, has similar robustness as the compared systems for decreased visual impact. Using the DWT, defined in JPEG 2000, this technique is readily applicable in the area of digital cinema, archiving and surveillance.

\section{ACKNOWLEDGMENT}

This research was supported by the Fund for Scientific Research Flanders (project G.0206.08 and the postdoctoral fellowship of Peter Schelkens) and the DaVinci project of the Interdisciplinary Institute for Broadband Technology (IBBT).

\section{REFERENCES}

[1] P. Schelkens, A. Skodras, and T. Ebrahimi, Eds., The JPEG 2000 Suite. Chichester, UK: John Wiley \& Sons, 2009.

[2] K. Solanki, N. Jacobsen, U. Madhow, B. Manjunath, and S. Chandrasekaran, "Robust image-adaptive data hiding using erasure and error correction," IEEE Trans. Im. Processing, vol. 13, no. 12, pp. 1627 -1639, 2004.

[3] M. Barni, F. Bartolini, and A. Piva, "Improved wavelet-based watermarking through pixel-wise masking," IEEE Trans. Im. Processing, vol. 10, no. 5, pp. $783-791,2001$.

[4] B. Chen and G. Wornell, "Quantization index modulation: A class of provably good methods for digital watermarking and information embedding," IEEE Trans. Inform. Theory, vol. 47, no. 4, pp. 1423$-1443,2001$.

[5] D. Bardyn, A. Dooms, T. Dams, and P. Schelkens, "Comparative study of wavelet based lattice qim techniques and robustness against awgn and jpeg attacks," in IWDW '09: Proceedings of the 8th International Workshop on Digital Watermarking. Berlin, Heidelberg: SpringerVerlag, 2009, pp. 39-53.

[6] M. Costa, "Writing on dirty paper (corresp.)," IEEE Trans. Inform. Theory, vol. 29, no. 3, pp. 439 - 441, 1983.

[7] A. Lewis and G. Knowles, "Image compression using the 2-d wavelet transform," IEEE Trans. Im. Processing, vol. 1, no. 2, pp. $244-250$, 1992.

[8] M. C. Davey and D. J. C. MacKay, "Reliable communication over channels with insertions, deletions, and substitutions," IEEE Trans. Inform. Theory, vol. 47, no. 2, pp. 687-698, 2001.

[9] J. A. Briffa, H. G. Schaathun, and S. Wesemeyer, "An improved decoding algorithm for the Davey-MacKay construction," in Proc. IEEE Intern. Conf. on Commun., Cape Town, South Africa, May 23-27, 2010.

[10] — , "Improved insertion/deletion correcting codes for large block sizes," IEEE Trans. Inf. Theory, submitted for publication, 2009.

[11] D. Divsalar and F. Pollara, "Turbo codes for deep-space communications," Jet Propulsion Laboratory, California Institute of Technology, TDA Progress Report 42-120, 1995.

[12] Z. Wang, A. Bovik, H. Sheikh, and E. Simoncelli, "Image quality assessment: from error visibility to structural similarity," IEEE Trans. Im. Processing, vol. 13, no. 4, pp. $600-612,2004$. 